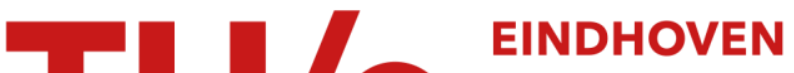 UNIVERSITY OF TECHNOLOGY
}

\section{Experimental evidence for a Mott-Wigner glass phase of magnetite above the Verwey temperature}

Citation for published version (APA):

Boekema, C., Lichti, R. L., Chan, K. C. B., Brabers, V. A. M., Denison, A. B., Cooke, D. W., Heffner, R. H., Hutson, R. L., \& Schillaci, M. E. (1986). Experimental evidence for a Mott-Wigner glass phase of magnetite above the Verwey temperature. Physical Review B: Condensed Matter, 33(1), 210-214.

https://doi.org/10.1103/PhysRevB.33.210

DOI:

10.1103/PhysRevB.33.210

Document status and date:

Published: 01/01/1986

\section{Document Version:}

Publisher's PDF, also known as Version of Record (includes final page, issue and volume numbers)

\section{Please check the document version of this publication:}

- A submitted manuscript is the version of the article upon submission and before peer-review. There can be important differences between the submitted version and the official published version of record. People interested in the research are advised to contact the author for the final version of the publication, or visit the $\mathrm{DOI}$ to the publisher's website.

- The final author version and the galley proof are versions of the publication after peer review.

- The final published version features the final layout of the paper including the volume, issue and page numbers.

Link to publication

\section{General rights}

Copyright and moral rights for the publications made accessible in the public portal are retained by the authors and/or other copyright owners and it is a condition of accessing publications that users recognise and abide by the legal requirements associated with these rights.

- Users may download and print one copy of any publication from the public portal for the purpose of private study or research.

- You may not further distribute the material or use it for any profit-making activity or commercial gain

- You may freely distribute the URL identifying the publication in the public portal.

If the publication is distributed under the terms of Article 25fa of the Dutch Copyright Act, indicated by the "Taverne" license above, please follow below link for the End User Agreement:

www.tue.nl/taverne

Take down policy

If you believe that this document breaches copyright please contact us at:

openaccess@tue.nl

providing details and we will investigate your claim. 


\title{
Experimental evidence for a Mott-Wigner glass phase of magnetite above the Verwey temperature
}

\author{
C. Boekema, R. L. Lichti, and K. C. B. Chan \\ Texas Tech University, Lubbock, Texas 79409 \\ V. A. M. Brabers \\ University of Technology, Eindhoven, NL-5600 MB Eindhoven, The Netherlands \\ A. B. Denison \\ University of Wyoming, Laramie, Wyoming 82071
}

\author{
D. W. Cooke, R. H. Heffner, R. L. Hutson, and M. E. Schillaci \\ Los Alamos National Laboratory, Los Alamos, New Mexico 87545
}

(Received 25 June 1985)

\begin{abstract}
New muon-spin-relaxation ( $\mu \mathrm{SR})$ results on magnetite are reported and discussed in light of earlier Mössbauer, neutron, and $\mu \mathrm{SR}$ results. Modification of the $\mu \mathrm{SR}$ anomaly (observed at $247 \mathrm{~K}$ in zero field), when an external magnetic field is applied, provides evidence that the anomaly results from cross relaxation between the muon Larmor precession and the electron-correlation process in the $B$ sublattice. The combined results strongly indicate that phonon-assisted electron hopping is the principal conduction mechanism above the Verwey transition temperature $\left(T_{V}\right)$. Together with theoretical evidence, these data support Mott's suggestion that above $T_{V}$ magnetite is in the Wigner-glass state.
\end{abstract}

Despite more than fifty years of magnetite research, no generally accepted explanation exists for the cause of the Verwey transition or for the conduction mechanism near room temperature (RT) ${ }^{1-9}$ Magnetite $\left(\mathrm{Fe}_{3} \mathrm{O}_{4}\right)$ is a mixed-valence oxide which undergoes a metal-to-insulator transition at the Verwey temperature $\left(T_{V} \approx 125 \mathrm{~K}\right){ }^{10}$ Neither the exact nature of this transition nor the complex behavior present between $T_{V}$ and RT is well understood. The main controversy has centered on the question of whether the "metallic" behavior observed above $T_{V}$ can best be explained using a collective electron-band model $^{4}$ or as phonon-assisted electron hopping, ${ }^{5-7,9}$ which can be considered as a narrow-polaron-band description. ${ }^{3}$

In 1979, Mott and others ${ }^{1}$ suggested that above $T_{V}$ magnetite should be considered as a Wigner glass, ${ }^{1-3}$ in which the extra $3 d$ electrons randomly occupy one-half of the iron $B$ sites. Due to the random fields produced by this disorder, each conduction electron (or small polaron) then occupies an Anderson-localized state. According to Mott, ${ }^{1-3}$ the electrical properties of such a glass are best described by a narrow polaron band. Nearest-neighbor phonon-assisted electron hopping then becomes the most probable mechanism for conduction.

Simultaneous condensation of the charge density and phonon density is responsible for the Verwey transition in the above model. ${ }^{7}$ Sharp discontinuities in thermal and transport properties such as conductivity and Seebeck coefficient indirectly support this picture. ${ }^{1,4,5}$ The interplay between lattice phonons and the charge carriers is important for both the modification of the charge density and the change in carrier motion which accompanies the phase transition. ${ }^{8}$ Presumably, this interaction is also re- lated to the various anomalies observed above $T_{V}$.

In this work we present the most direct experimental evidence to date for the existence of the MottWigner-glass state above $T_{V}$ in $\mathrm{Fe}_{3} \mathrm{O}_{4}$, and for the correctness of the narrow-polaron-band description of conduction in that phase. Our latest muon-spinrelaxation $(\mu S R)$ measurements strongly support the picture of hopping conduction. Supporting evidence from diffuse neutron scattering and Mössbauer data are also briefly discussed.

In a recent paper $^{11}$ we reported $\mu$ SR data describing positive-muon behavior in magnetite. Anomalies in the muon hyperfine interactions have been observed at $T_{V}$ and at $247 \mathrm{~K}$ in zero applied magnetic field. New results presented here indicate that the underlying mechanism of the anomaly at $247 \mathrm{~K}$ is probably associated with the dynamics of the phonon-assisted electron-conduction process in magnetite.

In the earlier $\mu \mathrm{SR}$ measurements of $\mathrm{Fe}_{3} \mathrm{O}_{4},{ }^{11}$ with increasing temperature the Verwey transition was clearly marked by a sudden increase in the muon hyperfine frequency and by a strong decrease in the muon relaxation rate. At $247 \mathrm{~K}$ a frequency decrease of equal magnitude, accompanied by a local maximum in the relaxation rate, was observed. This anomaly does not show any thermal hysteresis and is at the highest temperature for which experimental anomalies related to the Verwey transition have been reported. Aragón et al. ${ }^{10}$ recently observed a change in initial permeability near $250 \mathrm{~K}$, with a large thermal hysteresis, but present only for cation-deficient samples. We have performed $\mu \mathrm{SR}$ measurements on the same highly stoichiometric samples in which they ob- 
served no effect. The $\mu \mathrm{SR}$ anomaly at $247 \mathrm{~K}$ is observed for those samples identical to that reported earlier. Thus, we conclude that the $\mu \mathrm{SR}$ and permeability anomalies are not identical, although the underlying physics may be related.

We have suggested ${ }^{11}$ that the $247-\mathrm{K}$ anomaly observed in zero external field may be due to cross relaxation produced when the muon Larmor frequency approximately equals the temperature-dependent hopping rate for the conduction electrons in the $B$ sublattice. This suggestion was supported by the agreement ${ }^{11}$ between the electronhop time estimated from the muon frequency at the anomaly and that estimated from Mössbauer data (see later discussion).

Our latest $\mu$ SR experiments were based upon the idea that if the cross-relaxation interpretation ${ }^{11}$ of the $247-\mathrm{K}$ anomaly is correct, a shift of the anomaly temperature would occur at higher external fields. Application of an external field will increase the muon frequency $\left(f_{\mu}\right)$, and thus tune the cross relaxation to a shorter electron-hop time at a higher temperature. Temperature sweeps at 2 and 3 kOe were performed below RT. In Figs. 1 and 2, results for the temperature dependence of $f_{\mu}$ and relaxation rate are depicted. As can be seen, a field-dependent shift in the anomaly temperature is observed. The field dependence near $T_{V}$, from measurements performed earlier, ${ }^{12}$ indicates that the jump in $f_{\mu}$ is reversed at $T_{V}$. For both nonzero fields there appear to be two peaks in the relaxation data (see Fig. 2): one at about $245 \mathrm{~K}$ and the second at the "cross-relaxation" temperature evident in Fig. 1.

In Fig. 3 we have plotted the inverse-temperature

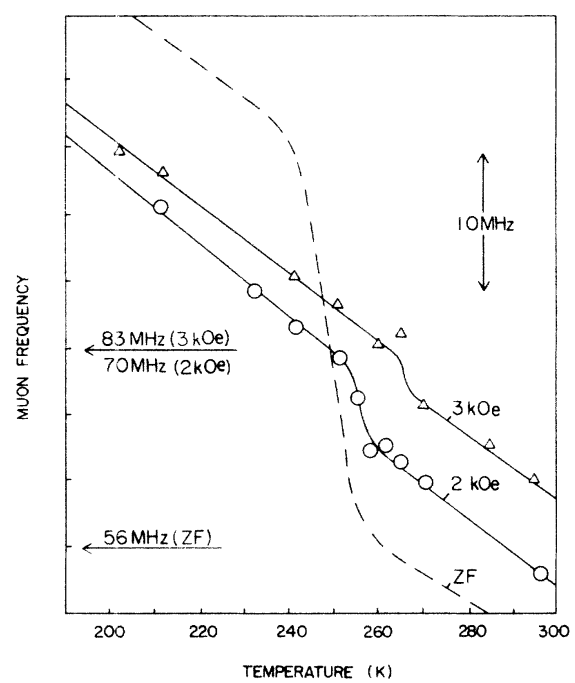

FIG. 1. Temperature dependence of the muon hyperfine frequencies at $0 \mathrm{kOe}$ (dashed line), $2 \mathrm{kOe}(\mathrm{O})$, and $3 \mathrm{kOe}(\triangle)$. Note the temperature shift in the frequency jump. The frequency zeros have been shifted for easy comparison purposes. The frequency division is $0.5 \mathrm{MHz}$ with the actual frequency value indicated at one point for each field. When one plots the local internal field [thus corrected for the effective (Ref. 11) external field] the three lines coincide at higher temperatures. For $B_{\text {ext }}=2$ (3) kOe the typical error bar is $0.15(0.25) \mathrm{MHz}$.

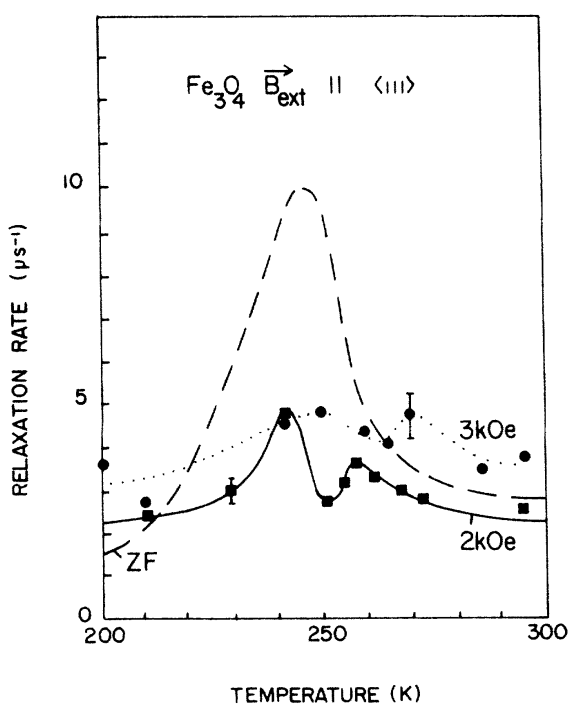

FIG. 2. Temperature dependence of the muon relaxation rate at 0-, 2-, and 3-kOe applied fields. For $B_{\text {ext }}=2$ (3) kOe the errors are $0.3(0.4) \mu \mathrm{s}^{-1}$. The curves are meant to guide the eye. The curve for zero field is taken from Ref. 11.

dependence of $f_{\mu}$ at which the anomaly takes place. An Arrhenius law fits well and yields an activation energy of $0.11 \mathrm{eV}$, which is identical to that obtained from conductivity measurements below $T_{V} \cdot^{5,6,13}$ The attempt frequency for electron-hopping motion obtained from our data is $\sim 5.6 \times 10^{10} \mathrm{~Hz}$. The $0.11 \mathrm{eV}$ may represent the barrier for nearest-neighbor hops with the conductivity near RT being additionally dependent on a second parameter related to the electron-phonon coupling. Single-parameter fits to conductivity in this temperature region yield much smaller values for a barrier, ${ }^{5}$ but this is then temperature dependent and should not, in general, be equated with activation energy for near-neighbor hops. Two-parameter fits consistent with a constant barrier near $0.1 \mathrm{eV}$ for local hopping motion have been successfully applied ${ }^{14}$ to the full range of conductivity measurements in magnetite.

In neutron-scattering measurements ${ }^{15,16}$ correlated

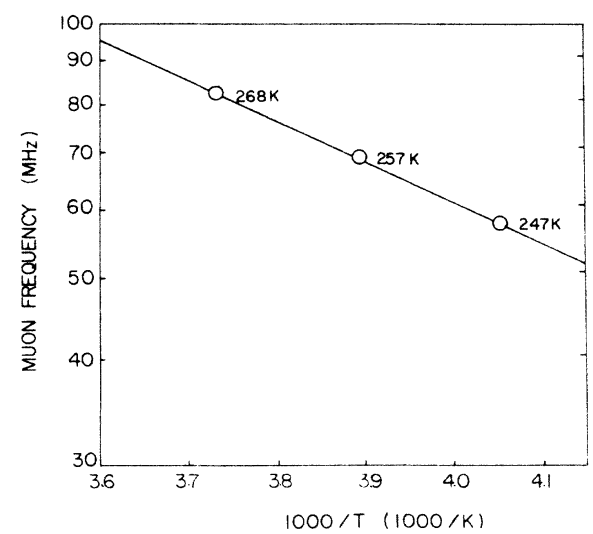

FIG. 3. The relationship between muon hyperfine frequency and inverse temperature, both at the point of cross relaxation. An Arrhenius-law fit gives a $0.11-\mathrm{eV}$ activation energy. 
motions of atomic groups have been indirectly observed. Fujii et al. ${ }^{15}$ observed spotlike scattering just above $T_{V}$. Shapiro et al. ${ }^{16}$ reported a different type of diffuse scattering, yielding diffuse streaks similar to patterns observed by electron diffraction. ${ }^{17}$ Both neutron results show apparent critical phenomena which disappear below $T_{V}$. The spotlike scattering observed between $T_{V}<T<T_{V}+5 \mathrm{~K}$ is associated with the structure of the low-temperature phase, whereas the diffuse scattering, observable to at least $200 \mathrm{~K}$, is closely related to atomic displacements.

The neutron diffuse-scattering results were later interpreted in terms of the formation of molecular polarons. ${ }^{1,18}$ In this picture, the Verwey phase transition can be viewed as a cooperative interaction of molecular polarons, leading to an ordering of $\mathrm{Fe}^{2+}$ and $\mathrm{Fe}^{3+}$ ions in the $B$ sublattice below $T_{V}$. Extrapolation of the experimental data $^{16}$ indicates that the molecular polarons cease to exist above about $250 \mathrm{~K}$.

As mentioned above, an independent estimate ${ }^{11}$ of the electron-hop time was obtained from Mössbauer studies in pure magnetite. ${ }^{19,20}$ In the Mössbauer studies a large change in the temperature gradient of the $B$-site linewidth $\left(\Gamma_{B}\right)$ and a sudden decrease for the $A$-site linewidth $\left(\Gamma_{A}\right)$, otherwise independent of temperature, was observed at about $240 \mathrm{~K}$. These observations are consistent with the notion that molecular polarons are formed below $250 \mathrm{~K}$.

The above conclusions regarding conduction-electron behavior have been drawn from pure-magnetite studies in which corrections have been made for the small magnetic anisotropy. These conclusions are in sharp contrast to the findings of Evans et al..$^{4,21}$ and van Diepen. ${ }^{22}$ The latter study shows that, for an applied magnetic field parallel to the $\langle 100\rangle$ axis, $\Gamma_{B}$ reduces nearly to $\Gamma_{A}$ at RT (see Table I). However, from the reported spectra, it cannot be said that all the broadening is due to magnetic anisotropy. Evans et al. ${ }^{4,21}$ found that above RT the temperature dependence of $\Gamma_{B}-\Gamma_{A}$ is explained by an increase of $\Gamma_{A}$ with increasing temperature instead of a decrease of $\Gamma_{B}$. In Table I temperature-dependent Mössbauer linewidth data from the conflicting studies are compared. Boekema et al. ${ }^{19,20}$ have found only a very slight increase, if any, in $\Gamma_{A}$ with temperature and unexpected larger $\Gamma_{A}$ broadening at higher temperatures in $A$-site doped magnetite.
In an attempt to interpret these $\mu \mathrm{SR}$ results in a manner consistent with the more indirect results of the neutron-scattering and Mössbauer studies in terms of one unified model, the assumption is made that at about 250 $\mathrm{K}$ molecular polarons begin to form as a result of smallpolaron pairing. With decreasing temperature the interactions between the small polarons, leading to the molecular-polaron formation, may, perhaps suddenly, increase the electron-hop time such that it crosses the muon Larmor-precession time. The cross-relaxation interpretation predicts an electron-correlation time of $2.8 \mathrm{~ns}$ which is in good agreement ${ }^{11}$ with the estimate of $3 \pm 1 \mathrm{~ns}$ from Mössbauer measurements.

The change in muon frequency at the anomaly is directly associated with the change in the magnetic environment of the muon site. The hop rate of the small polarons is significantly faster than the muon precession frequency above the "cross-relaxation" temperature but is slower below this temperature due to polaron pairing. The local lattice distortions associated with the conduction electron will complicate matters due to the fact that the effective field at the muon site is modified by atomic displacements as well as changes in the electron-hop rate.

The apparent occurrence of two maxima in the muon relaxation rate is consistent with two phenomena. The peak near $250 \mathrm{~K}$ in each temperature sweep (see Fig. 2) would thus be due to molecular-polaron formation, while the one at the anomaly temperature is the cross-relaxation effect discussed above.

The observed activation energy of $0.11 \mathrm{eV}$ may be related to an average energy barrier in the electron-hop process at temperatures above the molecular-polaron-formation temperature near $250 \mathrm{~K}$. According to Ihle and Lorenz, ${ }^{9,23}$ in the temperature range above $T_{V}$ there is a large number of electronic states showing short-range order in the $B$ sublattice. Molecular polarons provide both the electron correlation and short-range order. A basic reason for the short-range order is the relatively strong nearest-neighbor Coulomb interaction energy $\left(U_{1}\right)$ between the "extra" $3 d$ electrons in the $B$ sublattice. Ihle and Lorenz give a theoretical value for $U_{1}$ of $0.11 \mathrm{eV}$. Short-range order should also yield a peak near $U_{1}$ in the optical conductivity of $\mathrm{Fe}_{3} \mathrm{O}_{4}$, because the corresponding charge-transfer excitations induced by photons correspond

TABLE I. Reported values of Mössbauer linewidths in $\mathrm{Fe}_{3} \mathrm{O}_{4}$ at different temperatures. (No corrections were made for the small magnetic anisotropy.)

\begin{tabular}{|c|c|c|c|c|}
\hline$T(\mathbf{K})$ & $\Gamma_{A}(\mathrm{~mm} / \mathrm{s})$ & $\Gamma_{B}(\mathrm{~mm} / \mathrm{s})$ & Sample & Reference \\
\hline RT & 0.280 & $0.413^{\mathrm{a}}$ & polycrystalline & Boekema et al. ${ }^{b}$ \\
\hline RT & 0.28 & 0.30 & $\begin{array}{c}\text { single crystal } \\
\mathbf{H}_{\text {ext }}||\langle 100\rangle\end{array}$ & van Diepen ${ }^{c}$ \\
\hline RT & 0.349 & 0.392 & polycrystalline & Evans et al. ${ }^{\mathrm{d}}$ \\
\hline 703 & 0.376 & 0.371 & polycrystalline & Evans et al. ${ }^{\mathrm{d}}$ \\
\hline 672 & 0.291 & 0.314 & polycrystalline & Boekema ${ }^{e}$ \\
\hline
\end{tabular}

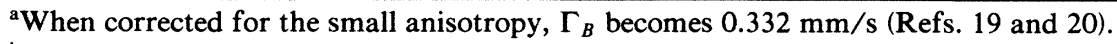

${ }^{\mathrm{b}}$ Reference 19.

${ }^{\mathrm{c}}$ Reference 22.

${ }^{\mathrm{d}}$ Reference 21.

${ }^{\mathrm{e}}$ Reference 20. 
to nearest-neighbor hops, requiring an excitation energy of about $U_{1}$ for each electron. ${ }^{23}$ In detailed optical measurements, Kuipers and Brabers ${ }^{6,13}$ found an absorption peak at $0.075 \mathrm{eV}$ for magnetite. Other arguments for electron correlations for $B$-site electrons are the occurrence of short-range charge (and spin) density oscillations found experimentally in Mössbauer studies, ${ }^{24}$ as well as theoretically, ${ }^{25}$ for slightly $B$-site doped magnetite above $T_{V}$. This is in contrast to results of other Mössbauer studies $^{4,21}$ which indicate that a collective electron-band description should be used. However, their results lean heavily on the method ${ }^{26}$ of Mössbauer data analysis.

A major remaining question in the interpretation of the $\mu \mathrm{SR}$ data on the $247-\mathrm{K}$ anomaly is the dependence of the magnitude of the jump in muon frequency on the applied field. Further, all of the applied-field data have been analyzed assuming a single frequency. However, within the model introduced above one might expect to observe two frequencies: one as observed at temperatures above the anomaly where the local fields probed by the muon are dominated by independent small polarons, and the second as observed well below the anomaly where the local fields are influenced by the short-range order, or molecular polarons. If one assumes a gradual pairing of the small polarons into the molecular polarons rather than a sudden "transition," then the intensities of the two frequencies would change gradually with temperature. Additional $\mu \mathrm{SR}$ experiments are planned to clarify these points and to trace out the anomaly for several additional field strengths.

Finally, our experimental results support the narrowband scheme for $\mathrm{Fe}_{3} \mathrm{O}_{4}$ as proposed by Ihle and
Lorenz. $^{9,23}$ Results of electrical-conductivity measurements and energy-band calculations indicate ${ }^{1-6,27}$ that the subbands are touching or may overlap, such that finite electron density exists at the Fermi energy. In this case, as proposed by Mott, ${ }^{1-3}$ a Wigner glass forms in which electrons near the Fermi energy are in Anderson-localized states induced by other localized electrons. Formation of molecular polarons appears to be a major ingredient in the interpretation of the observed cross-relaxation anomaly at $247 \mathrm{~K}$ in zero applied field. Results of narrow-polaronband calculations and deductions from them are consistent with our findings. The combined $\mu \mathrm{SR}$, neutron scattering, and Mössbauer data can be interpreted as evidence for a Mott-Wigner-glass phase above $T_{V}$ in magnetite. Moreover, these results strongly support the idea of phonon-assisted electron hopping above the Verwey transition in magnetite.

The authors are grateful to Dr. J. M. Honig, Dr. C. J. Sandberg, and Dr. H. Harrison (Department of Chemistry and Central Crystal Growth Facility, Purdue University) for providing us the highly stoichiometric magnetite crystals. We thank especially Dr. J. M. Honig for stimulating discussions. The research at Los Alamos is supported by the U. S. Department of Energy; the research at Texas Tech is supported partly by the Robert A. Welch Foundation. Two of the authors (C.B. and R.L.L.) wish to thank Mary C. Baker and other members of the condensedmatter division (Physics Department, Texas Tech University) for their stimulating interest and discussions. Two of the authors (C.B. and V.A.M.B) acknowledge a North Atlantic Treaty Organization (NATO) travel grant.
${ }^{1}$ Proceedings of the International Meeting on Magnetite and Other Materials Showing a Verwey Transition, Cambridge (1979) [Philos. Mag. B 42, No. 3 (1980)].

${ }^{2}$ N. F. Mott, Festkoerperprobleme 19, 331 (1979).

${ }^{3}$ N. F. Mott, Metal-Insulator Transitions (Taylor and Francis, London, 1974).

${ }^{4}$ B. J. Evans, in Magnetism and Magnetic Materials-1974 (San Francisco), proceedings of the 20th Annual Conference on Magnetism and Magnetic Materials, edited by C. D. Graham, G. H. Lander, and J. J. Rhyne (AIP, New York, 1975), p. 73.

5 J. M. Honig, Solid State Chem. 45, 1 (1982).

${ }^{6}$ A. J. M. Kuipers and V. A. M. Brabers, Phys. Rev. B 14, 1401 (1976); 20, 594 (1979).

7J. B. Sokoloff, Phys. Rev. B 5, 4496 (1972); 13, 2003 (1976).

${ }^{8}$ B. Lorenz and D. Ihle, Phys. Status Solidi B 96, 659 (1979); B.

K. Chakraverty, Philos. Mag. B 42, 473 (1980).

${ }^{9}$ D. Ihle, Phys. Status Solidi B 121, 217 (1984); D. Ihle and B. Lorenz, J. Phys. C 18, L647 (1985).

${ }^{10}$ R. Aragón, D. J. Buttrey, J. P. Shepherd, and J. M. Honig, Phys. Rev. B 31, 430 (1985); R. Aragón, J. P. Shepherd, J. W. Koenitzer, D. J. Buttrey, R. J. Rasmussen, and J. M. Honig, J. Appl. Phys. 57, 3221 (1985).

${ }^{11}$ C. Boekema, R. L. Lichti, V. A. M. Brabers, A. B. Denison, D. W. Cooke, R. H. Heffner, R. L. Hutson, M. Leon, and M. E. Schillaci, Phys. Rev. B 31, 1233 (1985). For more informa- tion on muon behavior in magnetic oxides, see A. B. Denison, J. Appl. Phys. 55, 2278 (1984); C. Boekema, Hyperfine Interact. 17-19, 305 (1984); C. Boekema, R. L. Lichti, and K. J. Rüegg, Phys. Rev. B 30, 6766 (1984).

${ }^{12}$ C. Boekema (unpublished).

${ }^{13}$ A. J. M. Kuipers and V. A. M. Brabers, Phys. Rev. Lett. 39, 488 (1977).

${ }^{14}$ R. Aragón, D. J. Buttrey, R. J. Rasmussen, and J. M. Honig, Bull. Am. Phys. Soc. 30, 275 (1985).

${ }^{15}$ Y. Fujii, G. Shirane, and Y. Yamada, Phys. Rev. B 11, 2036 (1975).

${ }^{16}$ S. M. Shapiro, M. Izumi, and G. Shirane, Phys. Rev. B 14, 200 (1976).

${ }^{17}$ K. Chiba, K. Suzuki, and S. Chikazumi, J. Phys. Soc. Jpn. 39, 839 (1975).

${ }^{18}$ Y. Yamada, N. Wakabayashi, and R. M. Nickbow, Phys. Rev. B 21, 4642 (1980).

${ }^{19}$ C. Boekema, J. de Jong, F. van der Woude, and G. A. Sawatzky, Physica 86-88B, 948 (1977).

${ }^{20}$ C. Boekema, Ph.D. thesis, University of Groningen, 1977.

${ }^{21}$ B. J. Evans, in Magnetism and Magnetic Materials-1972 (Denver), proceedings of the 18th Annual Conference on Magnetism and Magnetic Materials, edited by C. D. Graham and J. J. Rhyne (AIP, New York, 1973), p. 1398; B. J. Evans and Hang Nam Ok, Physica 86-88B, 931 (1977); Hang Nam 
Ok, Lu Su Pan, and B. J. Evans, Phys. Rev. B 17, 85 (1978).

${ }^{22}$ A. M. van Diepen, Phys. Lett. 57A, 354 (1976).

${ }^{23}$ D. Ihle and B. Lorenz, Philos. Mag. B 42, 337 (1980); D. Ihle, Z. Phys. B 58, 91 (1985).

${ }^{24}$ C. Boekema, F. van der Woude, and G. A. Sawatzky, J. Phys. C 9, 2439 (1976).

${ }^{25}$ D. Ihle and B. Lorenz, J. Phys. C 10, 1473 (1977).

${ }^{26}$ In this method, additivity of the changes in the hyperfine fields due to impurities within neighboring shells is used.
This additivity assumption is invalid as has been shown in a Mössbauer study [C. Boekema, P. C. Jonker, G. Filoti, F. van der Woude, and G. A. Sawatzky, Hyperfine Interact. 7, 45 (1979)] of slightly doped lanthanum-orthoferrite.

27J. R. Cullen, B. Chaudhury, and E. Callen, Phys. Lett. 38A, 113 (1973); J. R. Cullen and E. R. Callen, Phys. Rev. B 7, 397 (1973); B. Lorenz and D Ihle, Phys. Status Solidi B 69, 451 (1975). 